\title{
Injection laryngoplasty as miniinvasive office-based surgery in patients with unilateral vocal fold paralysis - voice quality outcomes
}

\author{
Ewelina M. Sielska-Badurek ${ }^{1}$, Maria Sobol ${ }^{2}$, Katarzyna Jędra ${ }^{1}$, Anna Rzepakowska ${ }^{1}$, Ewa Osuch-Wójcikiewicz ${ }^{1}$, \\ Kazimierz Niemczyk ${ }^{1}$ \\ ${ }^{1}$ Department of Otolaryngology, Medical University of Warsaw, Warsaw, Poland \\ 2Department of Biophysics and Human Physiology, Medical University of Warsaw, Warsaw, Poland
}

Videosurgery Miniinv 2017; 12 (3): 277-284

DOI: https://doi.org/10.5114/wiitm.2017.68868

\begin{abstract}
Introduction: Injection laryngoplasty (glottis augmentation) is the preferred method in surgical management of unilateral vocal fold paralysis (UVFP). Traditionally, these procedures are performed in the operating room. Nowadays, however, these procedures have moved into the office.

Aim: To evaluate the voice quality after transoral injection laryngoplasty under local anaesthesia in patients with unilateral vocal fold paralysis.

Material and methods: Fourteen subjects ( 5 women and 9 men) with unilateral vocal fold paresis ( 9 with right vocal fold paresis and 5 with left vocal fold paresis) were included in the study. The mean age of the group was 57.8 \pm 19.0 years (32-83 years). All of the injection laryngoplasties were performed transorally, under local anaesthesia. The injection material was calcium hydroxylapatite. Before and 1, 3 and 6 months after the procedure the following variables were evaluated: voice perception, videostroboscopy, acoustic analysis, aerodynamic evaluation, and the subjective rating of the voice quality by the patient.

Results: After injection laryngoplasty, complete glottal closure was achieved or there was a significant improvement in the glottal closure of each subject. We noted great improvement in the post-injection objective and subjective voice outcomes and patients reported improvement in the voice-related quality of life.

Conclusions: The transoral approach for injection laryngoplasty under local anaesthesia is an effective and safe way to treat incomplete glottal closure in patients with UVFP. The transoral approach is an efficient alternative to other surgical techniques used for vocal fold injection.
\end{abstract}

Key words: phonosurgery, injection laryngoplasty, glottal insufficiency, vocal fold paralysis, calcium hydroxylapatite, transoral approach.

\section{Introduction}

Injection laryngoplasty (glottis augmentation) is the preferred method in surgical management of unilateral vocal fold paralysis (UVFP), vocal fold atrophy, vocal fold scar, sulcus vocalis, abductor spasmodic dysphonia and even in the treatment of stubborn laryngeal granulomas [1, 2]. Traditionally, these procedures are performed in the operating room [2, 3]. Nowadays, however, these procedures have moved into the office because of the improved endoscopes, local anaesthetic techniques and de-

\section{Address for correspondence}

Dr. Maria Sobol, Department of Biophysics and Human Physiology, Medical University of Warsaw, 5 Chałubińskiego St, 02-004 Warsaw, Poland, phone: +48 2262863 34, fax: +48 2262878 46, e-mail: maria.sobol@wum.edu.pl 
velopment of different injectable systems. Glottis augmentation under local anaesthesia has been demonstrated to be a safe and clinically comparable alternative to injection laryngoplasty under general anaesthesia [4-6].

Injection laryngoplasty as an office-based procedure can be performed through different anatomical approaches: through the thyroid cartilage, transcricothyroid membrane, transthyrohyoid membrane or transorally, while visualizing the larynx with an endoscope. The transoral approach is a less invasive approach which is carried out through the mouth.

Because of the increase in population ages, as well as some of the most common causes of UVFP (e.g. thyroid surgery, cervical spine surgery), we can expect the incidence of UVFP to escalate as well [7-10]. The most common aetiology of vocal fold paralysis is iatrogenic; therefore UVFP most often occurs secondarily to complications from surgery, intubation, or both [7, 10-12]. Essentially, patients with UVFP often acquire multiple morbidities including general health concerns and psychosocial concerns. Consequently, there is a growing need to avoid general anaesthesia in procedures of these patients. Another advantage of the procedure is cost-effectiveness [13].

Although there is a steady growth of interest in awake injection laryngoplasty, the only data published are the outcome of transcutaneous glottis augmentation or, as in one study, the voice quality outcomes after injections from different approaches $[10,13-16]$. Until now there is no study presenting

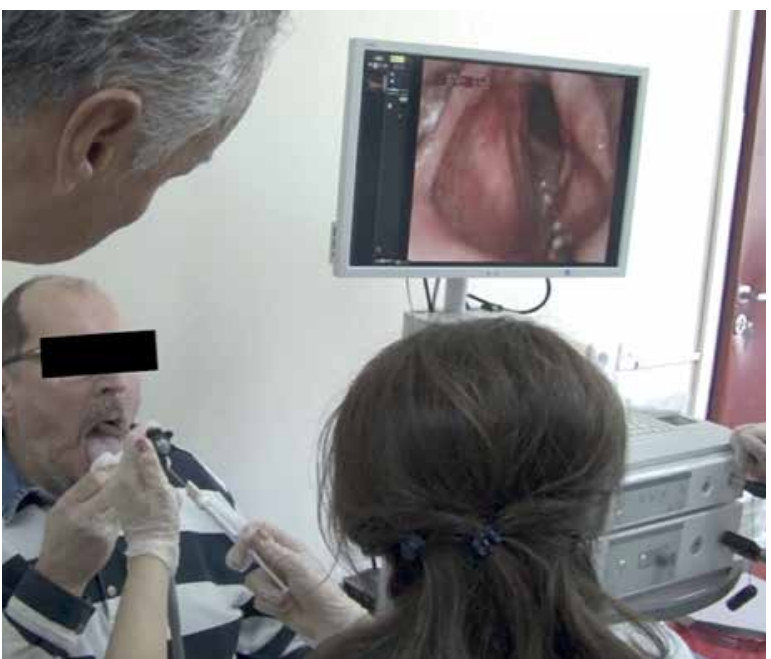

Photo 1. Phoniatrist and patient positioning for transoral vocal fold injection the results solely of transoral injection laryngoplasty under local anaesthesia, which is the less invasive approach.

\section{Aim}

The objective of the study was to evaluate voice quality after transoral injection laryngoplasty under local anaesthesia in patients with unilateral vocal fold paralysis.

\section{Material and methods}

Approval was obtained from the Polish Local Ethics Committee of the Medical University of Warsaw.

\section{Participants}

Fourteen patients from our Phoniatrics Clinic ( 5 women and 9 men) with unilateral vocal fold paresis were included in the study. The mean age of the group was $57.8 \pm 19.0$ years, the median 62.5 years, and the range $32-83$ years. Nine subjects had right vocal fold paresis and $5 \mathrm{e}$ had left vocal fold paresis. In 13 patients the vocal fold paresis was associated with surgery $(9$ - thyroid surgery, 1 - carotid endarterectomy, 1 - neck paraganglioma surgery, 1 - thoracic surgery and 1 - skull base surgery) and in 1 subject with idiopathic etiology.

\section{Procedure}

All 14 injection laryngoplasties were performed transorally, under local anaesthesia (Photo 1). The injection material was calcium hydroxylapatite.

After local anaesthesia with topical lidocaine spray and lidocaine drip applied onto the base of the tongue, the posterior pharyngeal wall, the supraglottis and the vocal folds the patient was asked to hold his tongue with gauze. Visualisation of the target area was obtained with a transoral rigid 70-angled scope connected to Xion's EndoStrobeE system, while injection of Radiesse was delivered via a custom curved needle within the paraglottic space of the targeted vocal fold. The first injection was performed in the middle of the paraglottic space. Afterwards the patient was asked to phonate. If the glottal closure was incomplete and the voice quality did not improve, the next injection was delivered laterally to the vocal process, in the posterior part of the paraglottic space. In order to achieve complete glottal closure, in 1 female subject a third injection 
was performed in the middle of the contralateral vocal fold's paraglottic space.

To assess the patient's voice quality, the evaluation protocol according to the European Laryngological Society (ELS) was performed before and 1, 3 and 6 months after injection laryngoplasty [17]. According to the ELS protocol the following components of voice quality need to be considered: perception, videostroboscopy, acoustic analysis, aerodynamic evaluation and subjective rating of the voice quality by the patient.

The perceptual speaking voice assessment was performed during a short conversation with the participant according to the GRBAS scale proposed by Hirano [18]. Each parameter of the scale: $G$ (grade of hoarseness), R (roughness), B (breathiness), A (asthenic voice) and $S$ (strained voice) was scored with a four-point grading scale $(0-$ normal or absence of deviance; 1 - slight deviance; 2 - moderate deviance; 3 - severe deviance).

Videostroboscopy, as the main clinical diagnostic tool, showed the type of glottal closure, as well as the regularity and symmetry of vocal folds' vibration and the quality of the mucosal wave. Videostrobokymography (VSKG) and phonovibrograms (PVG) obtained using DiagnoScope software by Diagnova enabled us to objectify the videostroboscopy evaluation.

For the acoustic analysis (objective and non-invasive measure of vocal function) each participant performed a recording of a phonation vowel [a] at comfortable pitch level and intensity. Later the recording was analysed by Computerized Speech Lab (CSL) on KAY Model 4150 with software: MDVP (Multi-Dimen- sional Voice Program) and a microphone by Shure type SM 48 which was placed $20 \mathrm{~cm}$ from the patient's lips. Four classical parameters were chosen for further analysis: FO (MDVP_FO), Jitter (MDVP_ Jitt), Shimmer (MDVP_Shim) and NHR (MDVP_NHR).

For the simplest aerodynamic parameter of voicing, as well as an objective and non-invasive measure of vocal function, the maximum phonation time (MPT) was performed and specified in seconds. The subject performed three trials of maximum prolongation of a vowel [a] at a spontaneous, comfortable pitch level and loudness after maximal inspiration. Each trial was recorded using the DIVAS Xion software. For further analysis, the longest maximum phonation time for the vowel [a] (MPTa) was selected.

For the self-assessment of voice quality, the Voice-Related Quality of Life (VRQoL) questionnaire was used, which was translated and validated as a reliable method of voice evaluation in the Polish population [19]. The overall score ranges from 10 to 50 , with a higher number representing poorer VRQoL.

Additionally, the vocal loudness (DIVAS_Loud) of a phonation vowel [a] at comfortable pitch level and intensity was assessed with DIVAS Xion software and the microphone headset was at a fixed distance from the participant's lips.

\section{Statistical analysis}

A statistical analysis was performed using the Statistica 12 package Medical University of Warsaw. Biographical information and results of the MDVP_FO,

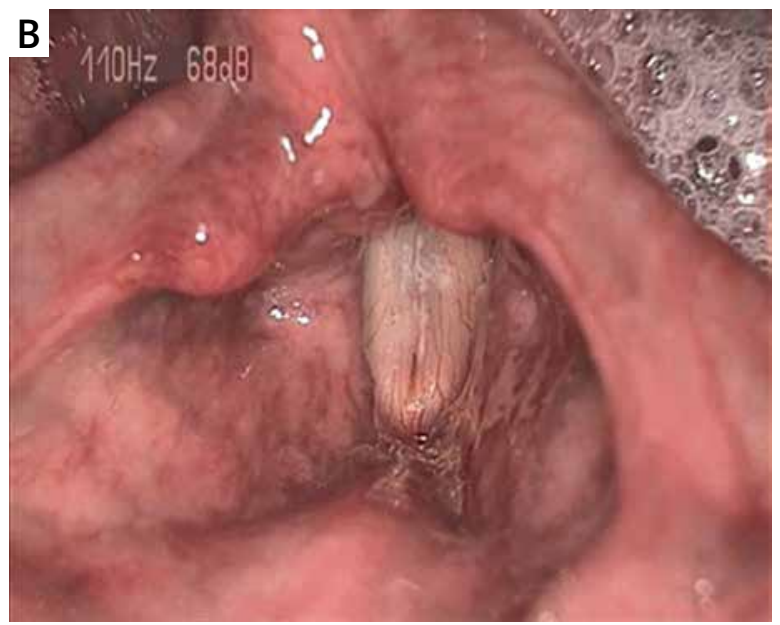

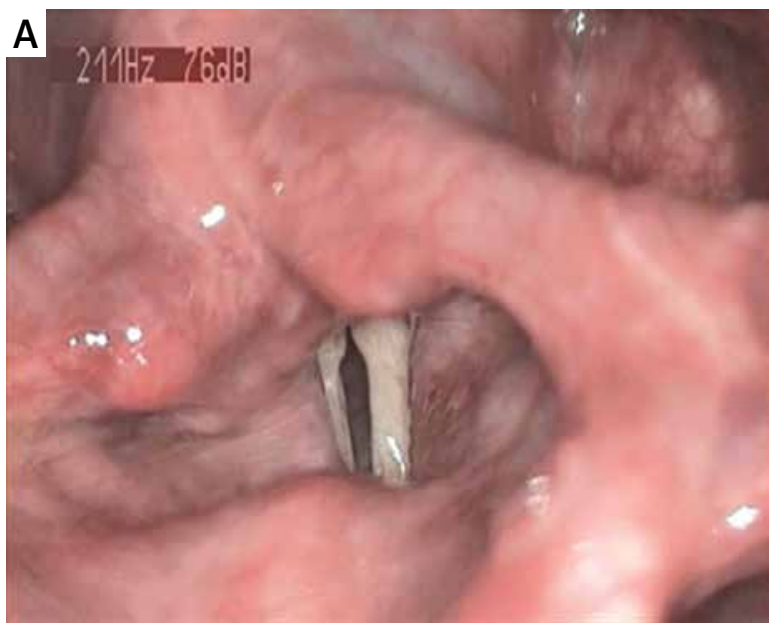

Photo 2. Videolaryngostroboscopy in patient no. 9. A - Videolaryngostroboscopic image of a left vocal fold paralysed in paramedian position with incomplete glottal closure. B - Videolaryngostroboscopic image after injection laryngoplasty 
MDVP_litt, MDVP_Shim, MDVP_NHR, MPTa, DIVAS Loud, G, R, B, A, S and VRQoL values, during each visit were summarized using descriptive statistics (mean, standard deviation, median and range). The nonparametric Wilcoxon pair test was performed to evaluate the differences between the first, second, third and fourth visit. Due to multiple comparisons Bonferroni corrections were used, $\alpha=0.017(\alpha=0.05 / 3)$.

\section{Results}

All participants scheduled for the injection could tolerate the procedure, therefore no procedure was

\section{A}

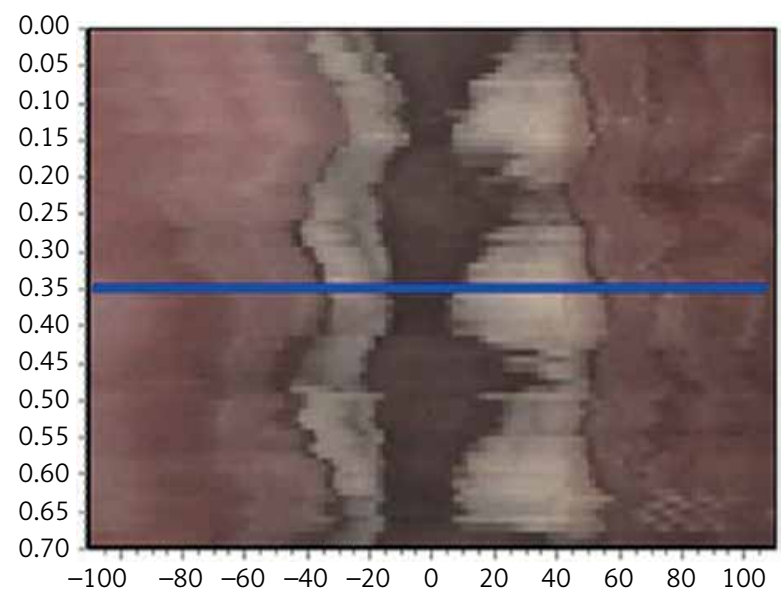

C

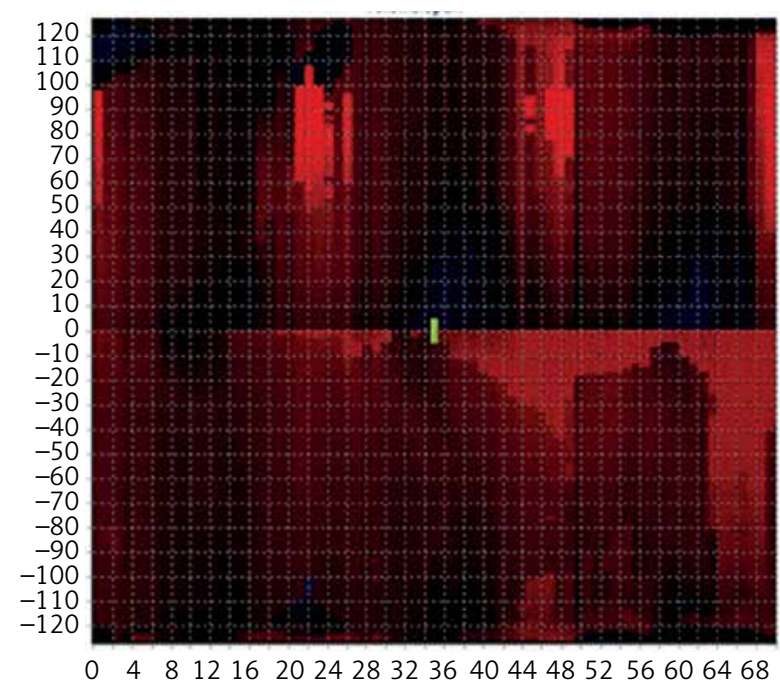

aborted. Thirteen participants left the hospital 2-4 $\mathrm{h}$ after the procedure. Only the first injected patient stayed at the hospital until the next day, although he did not demonstrate any side effect symptoms after the procedure. Thirteen patients did not need to take a lying position after the transoral injection laryngoplasty under local anaesthesia. Only 1 patient, who presented anxiety during the whole procedure, felt dizzy just after laryngoplasty. Following $4 \mathrm{~h}$ of observation in the clinic she was discharged home with no symptoms of dizziness.

After the injection laryngoplasty a complete glottal closure or significant improvement in glottal

\section{B}

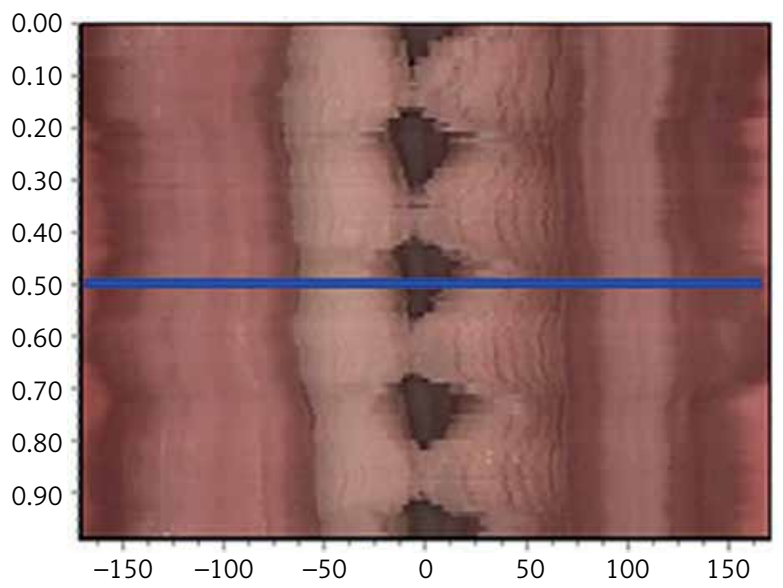

D

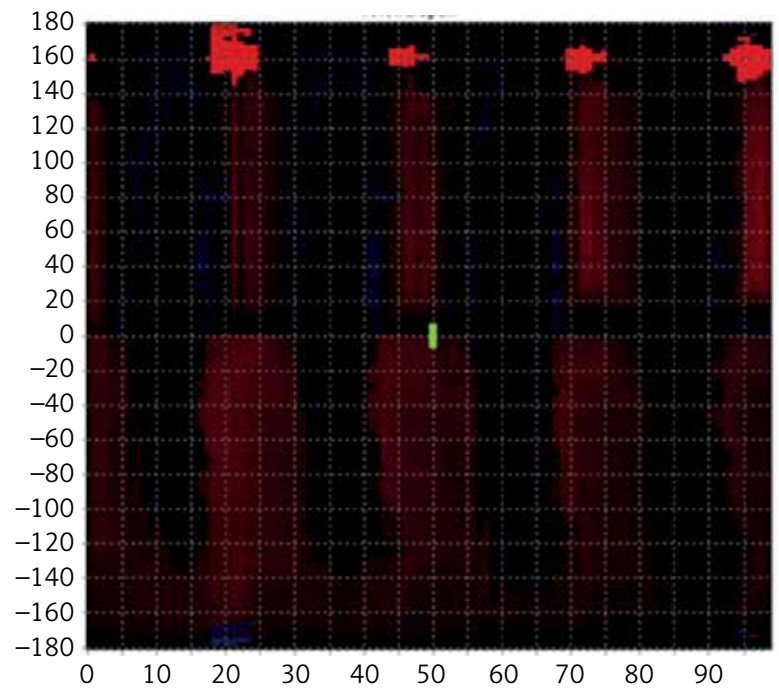

Photo 3. Visualization of the videostroboscopy: videostrobokymography (A, B) and phonovibrogram (C, D) in patient no. 9. A, C - Videostrobokymography section made from the median part of the glottis and phonovibrogram in left vocal fold paralysis. B, D - Videostrobokymography section made from the medial part of the glottis and phonovibrogram after injection laryngoplasty. In phonovibrograms: red colour expresses openings of the vocal folds during the vibration cycles, black colour expresses closing of the vocal folds during vibration cycles 
A

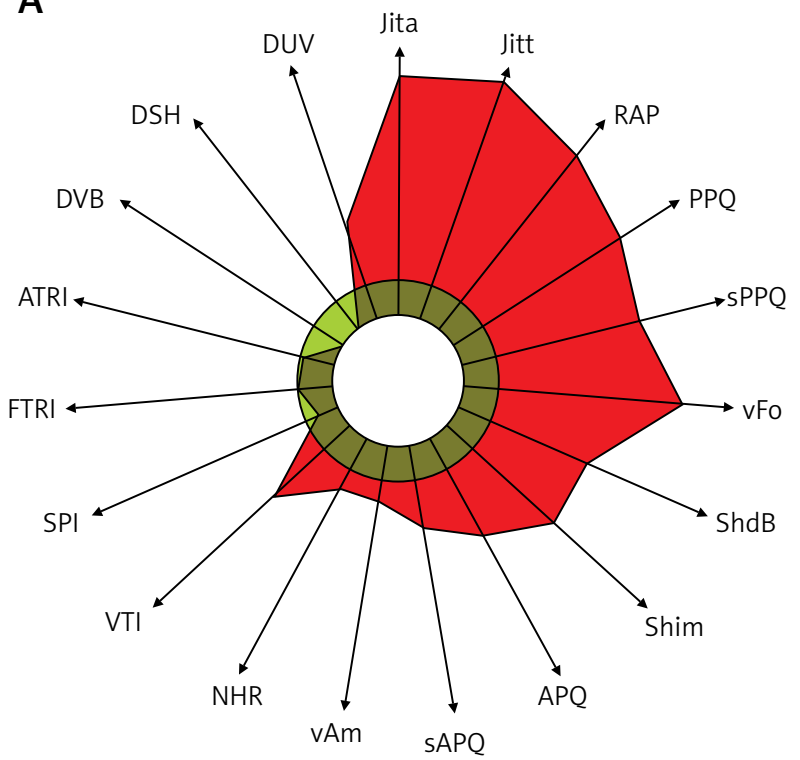

B

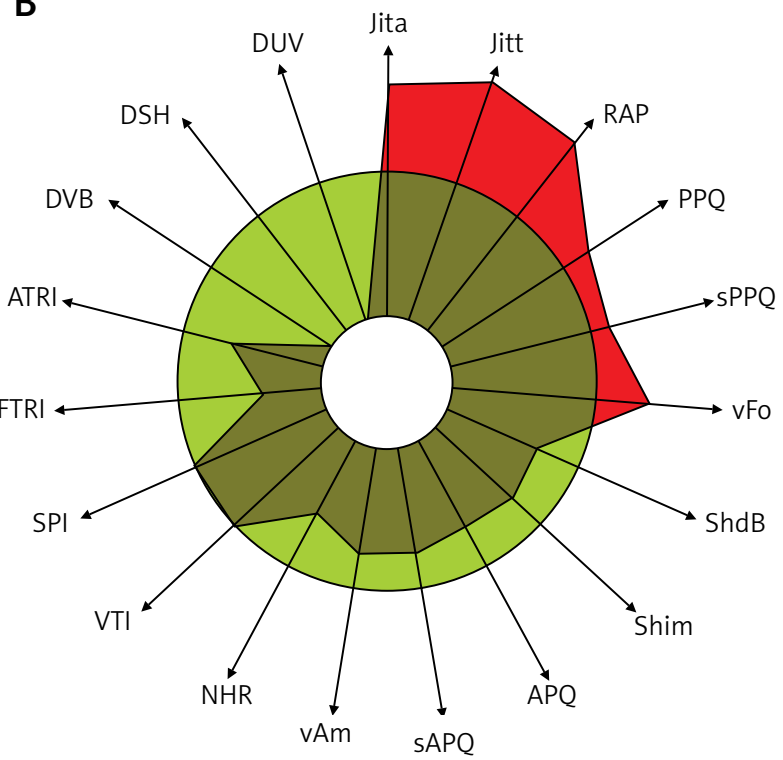

Figure 1. Acoustic analysis in patient no. 9. A - MDVP before injection laryngoplasty with evidences of severe perturbations in frequency and amplitude. B - MDVP after injection laryngoplasty, which underlines mild perturbations in frequency

closure in each subject (Photos 1-3, Figure 1) was achieved. Therefore, the participants' voice quality improved in each evaluated parameter. Table I shows the mean value, standard deviation (SD) and range of the objective parameters: acoustic analysis (MDVP_FO, MDVP_jitt, MDVP_Shim, MDVP_NHR), aerodynamic evaluation (MPTa), vocal loudness (DIVAS_Loud); and subjective: G, R, B, A, S and VRQoL values measured before and 1,3 and 6 months after injection laryngoplasty.

The acoustic analysis parameters improved almost by 100\% 1 month after injection, but the greatest improvement compared to the initial assessment for MDVP_Shim and MDVP_NHR was observed 3 months after surgery. Figure 2 provides the distribution of the MDVP_Shim values before and after injection laryngoplasty.

Similarly with aerodynamic evaluation and vocal loudness, the greatest prolongation of MPTa and increase in loudness value were noted 3 months after injection. The distribution of MPTa before and after surgery is given in Figure 3.

Each parameter of perceptual speaking voice assessment improved after injection laryngoplasty. However, statistically significant differences were found only between the $1^{\text {st }}$ (before) and $2^{\text {nd }}$ visit (1 month after injection) for the $\mathrm{G}$ and $\mathrm{S}$ parameters from the GRBAS scale.
A decrease in the VRQoL measure showed improvement in self-assessment of voice quality after injection laryngoplasty. Figure 4 provides the distribution of the VRQoL results before and after surgery.

\section{Discussion}

Injection laryngoplasty administered transorally under local anaesthesia proved to be an effective treatment method in patients with UVFP. Similarly to other studies reporting various surgical techniques for vocal fold injection, we observed great improvement in post-injection objective and subjective voice outcomes and patients reported improvement in voice-related quality of life $[2,3,13,15,16,20]$.

The great improvement in all assessed parameters can be explained by the excessive influence of glottal closure efficiency on voice. Our findings are comparable to those of Woo et al., who performed injection laryngoplasty transcutaneously [15]. They reported improvement of jitter, shimmer and NHR, 3 months after the procedure was performed through the thyrohyroid approach $(3,65 ; 7,45 ; 0,18$; respectively) [15], which is very similar to our results. In our study we achieved longer MPTa after injection compared to the results of Woo et al.: $12.2 \mathrm{~s}$ and 6.86-8.35 s, respectively [15]. Concerning the VRQoL measure value after 1 month, in this trial there was an improvement 


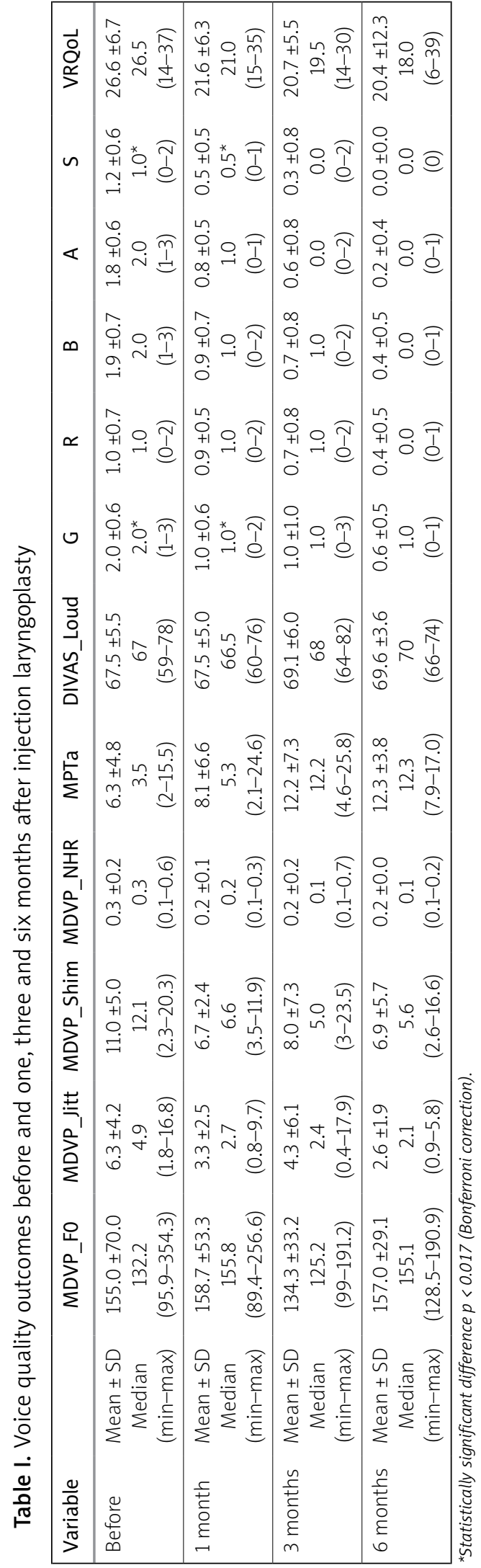

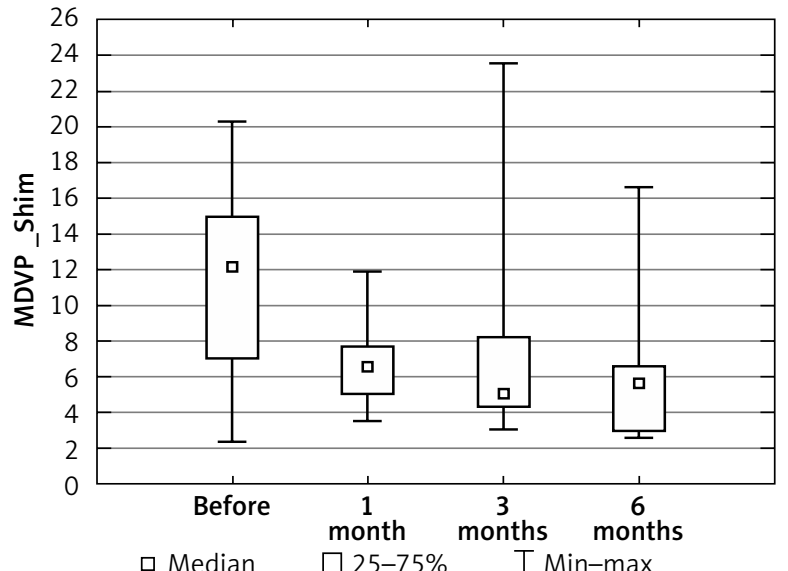

Figure 2. Box-whisker plot of the distributions of Shimmer from MDVP before, 1, 3 and 6 months after surgery

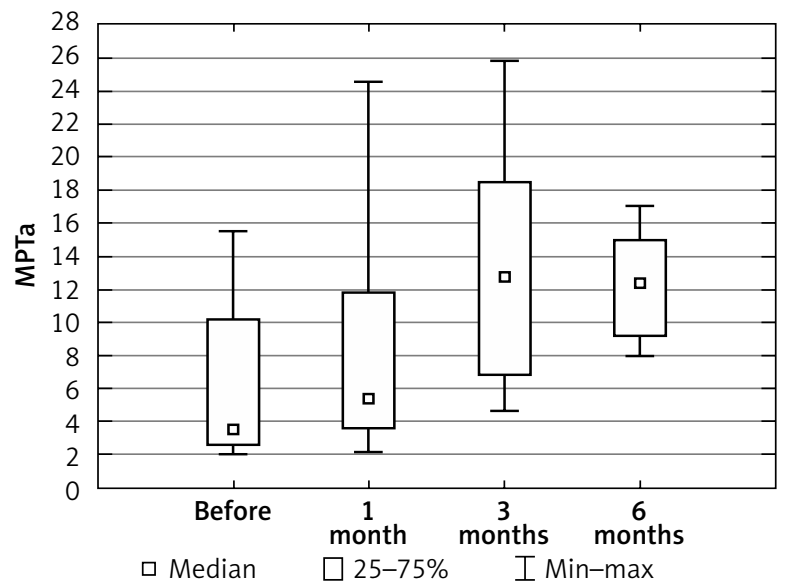

Figure 3. Box-whisker plot of the distributions of MPTa before, 1,3 and 6 months after injection laryngoplasty

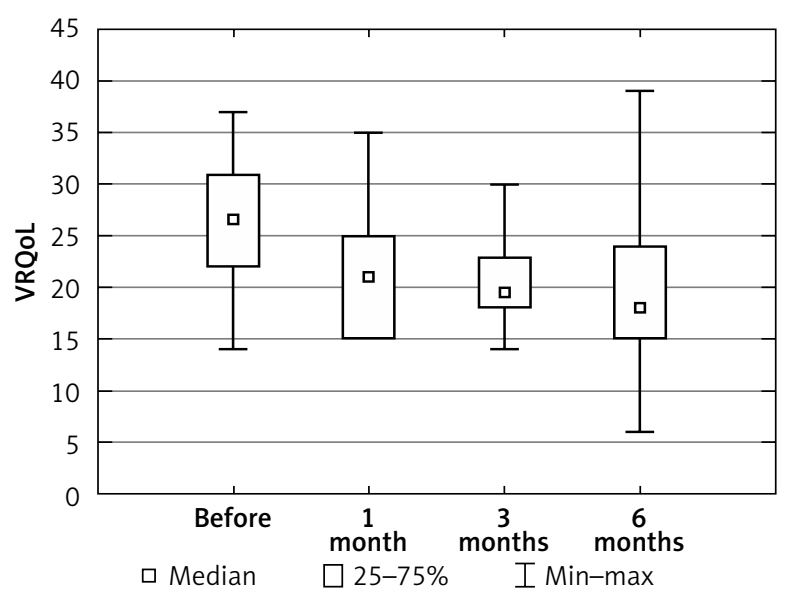

Figure 4. Box-whisker plot of the distributions of VRQoL before, 1, 3 and 6 months after injection laryngoplasty 
to 21.0, which corresponds to the result of Mathison et al.: 17.75 [20]. Mathison et al. used the transthyroid and transthyrohyoid procedure [20].

The great value of this study is the evaluation of voice outcomes with the full diagnostic protocol of ELS, while most other studies' authors assessed voice quality by using single diagnostic tools [2, 3, 13-15, 20]. Until now, only Jang et al. have evaluated voice outcomes with the full diagnostic protocol [16]. Jang et al. performed injection laryngoplasty percutaneously through the cricothyroid approach. Compared to their findings, we obtained longer MPTa (7,93 vs. 12,2; respectively) [16], which could relate to better glottis closure after the procedure.

Rosen and Thekdi, in 2004, in their trial of 11 terminal patients with UVFP, compared two different injection techniques: endoscopic direct vocal fold injection and percutaneous vocal fold injection [3]. Although the endoscopic direct vocal fold injection administered transorally was performed in the operating room with minimal sedation, they preferred this technique for accuracy and reliability. They underlined that placing the implant in the correct location was more difficult using the percutaneous method [3]. The current study supports the idea that the transoral approach facilitates precise placement of calcium hydroxylapatite, which is very useful in early experience of the technique.

Correspondingly to other minimal invasive surgical treatment methods, transoral injection laryngoplasty under local anaesthesia has proved to be a safe and cost-effective method [21-23]. According to Bove et al., the average reimbursement for injection laryngoplasty under general anaesthesia was \$ 2505 and \$ 496 for office-based injection laryngoplasty videosurgery [21], which showed the financial effectiveness of the second approach procedure. In this study 13 patients were discharged home on the day of the procedure, which significantly reduced hospital charges.

Although our first 14 injected patients with UVFP underwent the injection laryngoplasty via the transoral approach successfully, some authors report failed procedures while performing them under local anaesthesia $[13,20]$. Powell et al. report that $4 \%$ of local anaesthetic patients have intolerance of the procedure, especially those with high anxiety and a brisk gag reflex [13]. Those patients were unable to tolerate the simultaneous use of a rigid endoscope with injection.

\section{Conclusions}

The transoral approach for injection laryngoplasty under local anaesthesia is an effective and safe way to treat incomplete glottal closure in patients with UVFP. Based on the results of the study, the transoral approach is an efficient alternative to other surgical techniques used for vocal fold injection.

\section{Acknowledgments}

The authors wish to acknowledge the invaluable assistance of Dr Raul M. Cruz and Prof. Krzysztof Izdebski, who contributed to performance of the first injection laryngoplasty under local anaesthesia in May 2015, in Poland.

\section{Conflict of interest}

The authors declare no conflict of interest.

\section{References}

1. Mallur PS, Rosen CA. Vocal fold injection: review of indications, techniques, and materials for augmentation. Clin Exp Otorhinolaryngol 2010; 3: 177-82.

2. Belafsky PC, Postma GN. Vocal fold augmentation with calcium hydroxylapatite. Otolaryngol Head Neck Surg 2004; 131: 351-4.

3. Rosen CA, Thekdi AA. Vocal fold augmentation with injectable calcium hydroxylapatite: short-term results. J Voice 2004; 18 : 387-91.

4. Ward PH, Hanson DG, Abemayor E. Transcutaneous teflon injection of the paralyzed vocal cord: a new technique. Laryngoscope 1985; 95: 644-9.

5. Filho PA, Carrau RL, Buckmire RA. Safety and cost-effectiveness of intra-office flexible videolaryngoscopy with transoral vocal fold injection in dysphagic patients. Am J Otolaryngol 2006; 27: 319-22.

6. Grant JR, Hartemink DA, Patel N, Merati AL. Acute and subacute awake injection laryngoplasty for thoracic surgery patients. J Voice 2008; 22: 245-50.

7. Rosenthal LH, Benninger MS, Deeb RH. Vocal fold immobility: a longitudinal analysis of etiology over 20 years. Laryngoscope 2007; 117: 1864-70

8. Davies L, Welch HG. Increasing incidence of thyroid cancer in the United States, 1973-2002. JAMA 2006; 295: 2164-7.

9. Patil PG, Turner DA, Pietrobon R. National trends in surgical procedures for degenerative cervical spine disease: 1990-2000. Neurosurgery 2005; 57: 753-8.

10. Verma SP, Dailey SH. Office-based injection laryngoplasty for the management of unilateral vocal fold paralysis. I Voice 2014; 28: 382-6.

11. Myssiorek D. Recurrent laryngeal nerve paralysis: anatomy and etiology. Otolaryngol Clin North Am 2004; 37: 25-44.

12. Sielska-Badurek E, Domeracka-Kołodziej A, Zawadzka R, Dębowska-Jarzębska E. Vocal fold paralysis in the Medical Univer- 
sity of Warsaw's Ambulatory of Phoniatry in years 2000-2011. Otolaryngol Pol 2012; 66: 313-7.

13. Powell J, Carding P, Birdi R, et al. Injection laryngoplasty in the outpatient clinic under local anaesthetic: a case series of sixty-eight patients. Clin Otolaryngol 2014; 39: 224-7.

14. Kwon TK, An SY, Ahn JC, et al. Calcium hydroxylapatite injection laryngoplasty for the treatment of presbylaryngis: long-term results. Laryngoscope 2010; 120: 326-9.

15. Woo SH, Son YI, Lee SH, et al. Comparative analysis on the ef ficiency of the injection laryngoplasty technique using calcium hydroxyapatite (CaHA): the thyrohyoid approach versus the cricothyroid approach. J Voice 2013; 27: 236-41.

16. Jang JY, Lee G, Ahn J, et al. Early voice rehabilitation with injection laryngoplasty in patients with unilateral vocal cord palsy after thyroidectomy. Eur Arch Otorhinolaryngol 2015; 272: 3745-50.

17. Dejonckere PH, Bradley P, Clemente P, et al. A basic protocol for functional assessment of voice pathology, especially for investigating the efficacy of (phonosurgical) treatments and evaluating new assessment techniques. Eur Arch Otorhinolaryngol 2001; 258: 77-82.

18. Hirano M. Clinical examination of voice. Springer, New York, USA 1981.

19. Sielska-Badurek E, Rzepakowska A, Sobol M, et al. Adaptation and validation of the voice-related quality of life measure into Polish. J Voice 2016; 30: 773.e7-12.

20. Mathison CC, Villari CR, Klein AM, et al. Comparison of outcomes and complications between awake and asleep injection laryngoplasty: a case-control study. Laryngoscope 2009; 119: 1417-23.

21. Bove MJ, Jabbour N, Krishana P, et al. Operating room versus office-based injection laryngoplasty: a comparative analysis of reimbursement. Laryngoscope 2007; 117: 226-30.

22. Szeliga J, Jackowski M. Minimally invasive procedures in severe acute pancreatitis treatment - assessment of benefits and possibilities of use. Videosurgery Miniinv 2014; 9: 170-8.

23. Wróblewski T, Kobryn K, Nowosad M, Krawczyk M. Surgical treatment of GERD. Comperative study of WTP vs. Toupet fundoplication - results of 151 consecutive cases. Videosurgery Miniinv 2016; 11: 60-6.

Received: 12.04.2017, accepted: 20.05.2017. 